\title{
Acompañamiento del enfermo: Una experiencia de aprendizaje sobre el significado de la enfermedad
}

\author{
Armando O rtiz P1, Juan Pablo Beca I ${ }^{1}$, Sofía P Salas ${ }^{2}$, \\ Francisca Browne L ${ }^{1 a}$, Camila Salas A ${ }^{1 b}$.
}

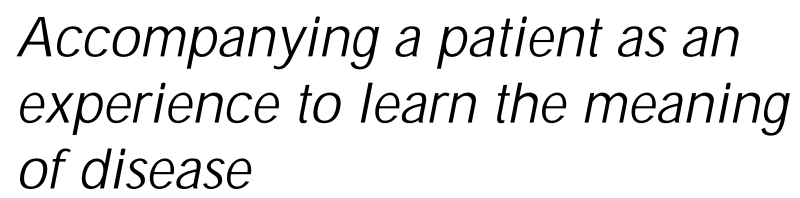

Background: Due to technical advances and progressive specialization, patient physician relationship evolved from being based on confidence and comprehension to be impersonal and contractual. Aim: To evaluate the change produced in medical students' concept of the meaning of disease, after living an experience of accompanying a patient and their family environment. Material and methods: We used a qualitative research methodology based on participative-action to evaluate an educational method in which a group of third year medical students accompanied a previously selected chronic patient during two months. We performed two focal groups, one previous to the experience and the second one a month later. Additionally, every student wrote a personal narration of the experience. We compared the students' perception of the meaning of disease for the patients and their relatives, before and after the experience. Results: The students confirmed their previous conception about chronic disease, but they also discovered the ways in which some patients and their families overcome their limitations. They pointed out their belief that the physician's comprehension of the meaning of disease for the patient and the family, a need to practice a good medicine and that this knowledge change the physician-patient relationship. Conclusions: This experience was an effective way of learning the meaning of disease, which might be a part of medical student' training, with the corresponding adjustments and changes (Rev Méd Chile 2008; 136: 304-9).

(Key words: Disease; Empathy; Students, medical)

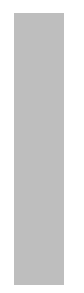

Recibido el 31 de julio, 2007. Aceptado el 16 de octubre, 2007.

Investigación financiada por Fundación Victor Grifols i Lucas de Barcelona, España y por la Dirección de Investigación de Universidad del Desarrollo.

${ }^{1}$ Centro de Bioética, Facultad de Medicina Clínica Alemana, Universidad del Desarrollo. Santiago de Chile. ${ }^{2}$ Departamento de Obstetricia y Ginecología, Escuela de Medicina, Pontificia Universidad Católica de Chile.

aSocióloga

${ }^{\mathrm{b}}$ Ayudante alumno

Correspondencia a: Dr. Armando Ortiz Pommier. Las Condes 12438, Lo Barnechea, Santiago-Chile. Fax: (562) 2999336.

E mail: aortiz@udd.cl 
$\mathrm{L}$ a medicina contemporánea, caracterizada por una creciente especialización, progreso tecnológico e incremento de costos, ha llevado a que el enfermo esté hoy mejor atendido en lo especializado y técnico, pero menos acompañado y comprendido en su realidad personal por parte del médico. La relación médico-paciente clásica, basada en mayor confianza y comprensión de parte del médico del significado de la enfermedad, ha dado paso a una relación más impersonal y contractual. Por su parte, el propósito de la enseñanza médica es formar médicos de excelencia científico-técnica, junto a una sólida formación ética y compromiso de servicio. Con este fin, se han introducido en el currículo cursos de bioética, psicología, comunicación, antropología y otras disciplinas de humanidades médicas. Sin embargo, estos cursos teóricos parecen insuficientes para que los estudiantes comprendan el significado de la enfermedad para el enfermo y su entorno socio-familiar, les falta ser testigos directos de la enfermedad en pacientes a quienes se conozca en lo personal. Ya que esto se logra difícilmente en la enseñanza de pregrado, parece necesario crear y probar formas de hacerlo.

La literatura muestra experiencias no evaluadas de acompañamiento prolongado de enfermos a nivel de postítulo ${ }^{1}$, y se propone incorporar precozmente a los estudiantes a la práctica clínica, con formas variadas de participación ${ }^{2}$. Se ha demostrado que los estudiantes tienen dificultades en sus primeros encuentros con pacientes, que rara vez comentan con sus docentes y que los que reciben formación en comunicación tienen mayor comprensión y competencia en decisiones compartidas $^{3,4}$. Por otra parte, la participación de pacientes como "educadores" tiene importantes beneficios para que los alumnos mejoren sus actitudes hacia ellos ${ }^{5}$. Sin embargo, no se ha evaluado de manera directa los cambios que se producirían en estudiantes de medicina sometidos a una experiencia vivencial con un "paciente tutor" previamente seleccionado, capaz de transmitir el significado profundo de vivir con una enfermedad, desde su perspectiva y su entorno familiar. Con este propósito, se diseñó una experiencia de acompañamiento a un enfermo, evaluando de manera cualitativa su efecto en la percepción de los estudiantes sobre el significado de la enfermedad.

\section{MATERIAL Y MÉTODO}

Se implementó y evaluó una experiencia vivencial, en la cual estudiantes de medicina se constituyeron en testigos y acompañantes de un enfermo por un período de dos meses, en los que sostuvieron uno a dos encuentros semanales con el enfermo y su familia. Para la evaluación se definió una metodología cualitativa de tipo investigación-acción participativa, que parte de la idea de que a través de la experiencia vivida se puede comprender la esencia del tema ${ }^{6}$. Se empleó técnica de Grupo Focal mediante dos entrevistas dirigidas por uno de los autores (FB), realizadas antes del inicio y un mes después de los encuentros con los pacientes. Además, cada estudiante escribió al término de cada encuentro una narración registrando, de manera anónima, sus reflexiones personales y datos de la historia del paciente. Durante el período de estudio se realizaron dos reuniones de los estudiantes con los investigadores para aclarar dudas y compartir experiencias. El proyecto fue aprobado por el Comité de Etica de la Universidad del Desarrollo.

Los estudiantes fueron alumnos de $3^{\text {er }}$ año de la Facultad de Medicina, Clínica Alemana Universidad del Desarrollo, que están iniciando su práctica clínica, a quienes se invitó de manera abierta. Luego de reuniones preliminares, se constituyó un grupo de 10 estudiantes, hombres y mujeres. La escasa disponibilidad horaria y el periodo de fin de año académico dificultaron constituir el grupo. Todos firmaron un documento de consentimiento informado. Un estudiante se retiró del estudio, por motivos personales, a dos semanas del inicio. En el primer grupo focal se evaluaron conocimientos y percepción sobre el significado de la enfermedad para una persona y su entorno familiar y social. Después recibieron un ciclo de sesiones preparatorias que incluyó la revisión de material bibliográfico relacionado con el tema y se analizaron las dificultades que podrían vivir en sus encuentros con los pacientes. Luego se asignó a cada alumno, aleatoriamente, un "paciente-tutor".

Los pacientes no fueron seleccionados por patologías sino por su cronicidad y su disponibilidad, fueron invitados por sus médicos tratantes y recibieron información detallada de parte de uno de los investigadores. Se invitaron hasta alcanzar 
un número de 10 enfermos voluntarios, quienes firmaron un documento de consentimiento informado y compromiso de colaboración. Se incluyó a 9 pacientes adultos con enfermedad crónica y a los padres de un niño con síndrome de Down. Los enfermos participantes tuvieron las características que se describen en la Tabla 1. Después del ciclo preparatorio, cada estudiante se puso en contacto con su paciente y lo visitó, sin el médico tratante, con un calendario de encuentros fijado entre enfermo y estudiante, incluyendo encuentros con miembros de la familia del paciente.

Las transcripciones de los grupos focales y las narraciones fueron analizadas mediante técnica de análisis de contenido. En los grupos focales se apreció cómo los estudiantes percibían la vivencia de la enfermedad para los pacientes y su entorno. La comparación de sus percepciones, antes y después de la experiencia, permitió establecer el efecto de esta experiencia en términos de aprendizaje y efectividad.

\section{Resultados}

Los alumnos manifestaron que su motivación para participar en este estudio fue conocer más profundamente la enfermedad y comprender su impacto en lo personal y familiar. En el grupo focal inicial expresaron que tener una enfermedad crónica significa limitaciones, pérdidas, frustración, angus- tia, incertidumbre, soledad, dedicación de tiempo al tratamiento de la enfermedad, tristeza, adaptación, conciencia de la diferencia, pérdida de autoestima, nuevo trato de parte de la familia y aislamiento, entre otras. Agregaron que también significa una oportunidad para superar situaciones adversas, lo cual para algunos puede ser fuente de satisfacción y de mayor unión familiar. Los alumnos se referían al significado de una enfermedad como "algo que hacía antes y ya no se puede hacer", "obliga a la persona a modificar sus quehaceres", "una especie de muleta", "un problema que lo limita", y "se sienten tristes". De lo anterior se desprende que para los estudiantes una enfermedad crónica significa limitación, necesidad de cambiar estilos de vida, adaptación y frustración. Es también un desafío, en la medida que la persona afectada se ve enfrentada a adaptarse a nuevas circunstancias, con éxito o fracaso, según las características psicológicas del paciente. Uno de los estudiantes expresó: "dependiendo de la persona, del apoyo de los demás, de su entorno y de su fuerza interior es como sobrellevan este proceso que lo puede llevar a cambiar definitivamente su estilo de vida, a protegerse un poco, y quizás a no poder superarlo". Antes de acompañar a sus respectivos pacientes, los alumnos comprendían que la problemática del enfermo afecta al grupo familiar, el cual también debe enfrentar cambios. La frase “...y después viene todo el problema para la familia, porque también

Tabla 1. C aracterísticas de los pacientes

\begin{tabular}{|c|c|c|c|}
\hline & Sexo & Edad & Diagnósticos principales \\
\hline 1 & M & 48 años & Cardiopatía severa, reflujo gastroesofágico y dependencia al tabaco \\
\hline 2 & $\mathrm{~F}$ & 70 años & $\begin{array}{l}\text { Hipertensión arterial, cirugía de recambio valvular, infarto cardíaco hace } \\
3 \text { años }\end{array}$ \\
\hline 3 & $\mathrm{~F}$ & 74 años & $\begin{array}{l}\text { Hemiplejia secundaria a accidente vascular cerebral, cáncer mamario } \\
\text { tratado }\end{array}$ \\
\hline 4 & $\mathrm{~F}$ & 3 meses & Síndrome de Down \\
\hline 5 & $\mathrm{~F}$ & 80 años & Estenosis aórtica severa, insuficiencia cardíaca \\
\hline 6 & M & 38 años & Diálisis por insuficiencia renal crónica \\
\hline 7 & M & 71 años & Secuelas de infarto cerebral \\
\hline 8 & $\mathrm{~F}$ & 64 años & Secuelas de hemorragia subaracnoídea, incontinencia, gran fumadora \\
\hline 9 & $\mathrm{~F}$ & 33 años & Epilepsia y trastorno depresivo \\
\hline
\end{tabular}


la familia tiene que cambiar la forma de operar en función de esta persona..." lo expresa con claridad. Frente a la familia, señalaron que el enfermo se siente "una carga" en cuanto no puede realizar sus rutinas y alguien tiene que hacerse cargo suyo: "Se sienten no solamente limitados por su condición sino que se sienten que son más molestia, aunque la familia no lo vea así, pero ellos se sienten así, como no independientes, más como una carga". En el primer grupo focal también manifestaron que la familia siente el deber de hacerse cargo del enfermo, de mantenerlo emocionalmente estable y de asegurar el cumplimiento del tratamiento. En este sentido, la familia asume el rol de "muleta" del paciente. A pesar de lo anterior, los estudiantes creen que la familia crece emocionalmente si se compromete con el enfermo, aunque en otros casos puede llevar a un quiebre o separación. Los participantes se situaron así imaginariamente frente a los significados de ser un enfermo crónico.

Después de la experiencia de acompañamiento, los estudiantes expresaron haber descubierto la forma positiva que tienen algunos pacientes para enfrentar su enfermedad, más allá de lo que esperaban encontrar. Observaron que algunos pacientes pueden sobrellevar su enfermedad de manera optimista, superando sus limitaciones, y que el abatimiento no es la situación general de los enfermos crónicos. Junto a lo anterior, los estudiantes percibieron cómo una enfermedad crónica afecta la autoestima en la medida que el enfermo deja de ser autovalente, y cómo la enfermedad dificulta el desempeño laboral. Los alumnos también expresaron haber percibido cómo la enfermedad crónica del paciente afecta a toda la familia. Observaron cómo un familiar asumía los roles del enfermo, situaciones en que el enfermo ya no aportaba como antes, familias que debieron vigilar permanentemente a una mujer con graves limitaciones, y a padres que debieron asumir un hijo con síndrome de Down. En la mayoría de los casos la familia tomó un rol de apoyo activo, pero en otros eran sólo testigos pasivos de la enfermedad. En casos excepcionales, la familia había casi abandonado al enfermo, con el sufrimiento y mayor menoscabo correspondiente.

En el segundo grupo focal los alumnos señalaron su convencimiento de que la comprensión del médico sobre el significado de la enfermedad para el paciente cambia su trato hacia el enfermo, permite adecuar el tratamiento y reforzar aspectos particulares de cada caso. Concluyeron que el médico necesita conocer la vida del paciente, su personalidad, sus valores, su capacidad y las condiciones ambientales y familiares para poder indicar y controlar bien el tratamiento. Los estudiantes manifestaron que conocer bien al enfermo y el significado de la enfermedad genera además una forma de crecimiento espiritual para el médico. Las siguientes frases apoyan estas ideas: “además del tratamiento, todo eso está bien, pero cuando uno ve que algo tiene una consecuencia positiva uno mismo tiene un sentimiento más intenso", o "porque el conocimiento uno lo tiene, pero el crecimiento personal no se logra si no se relaciona bien con los pacientes".

Analizando comparativamente la imagen que tenían los alumnos antes y después de esta experiencia de aprendizaje, se observó que su visión sobre cómo una enfermedad crónica afecta al enfermo y su entorno fue confirmada, tanto por su impacto negativo en la autonomía del paciente como en su variación en cada caso particular. Descubrieron además que algunos enfermos crónicos o sus familias tienen fortalezas que les permitieron superar limitaciones de manera más positiva de la que ellos imaginaban. Más que nuevos hallazgos, los alumnos reafirmaron conceptos previos y tomaron conciencia de la importancia de comprender globalmente la enfermedad como un elemento necesario para ejercer una buena medicina.

\section{DisCUSIÓN}

La formación de los estudiantes de medicina hace necesario que ellos adquieran, junto a los conocimientos científicos, determinadas actitudes y un concepto global sobre la enfermedad y su significado para las personas enfermas ${ }^{6}$. Adquirir conocimientos no implica por sí mismo cambios de actitud en los estudiantes ${ }^{7}$, es necesario encontrar métodos docentes que cumplan con estos objetivos. Una manera de hacerlo, además de la importancia formativa del currículo oculto ${ }^{8}$, puede ser la incorporación de experiencias con enfermos que sean instructores de alumnos y programas que incluyan un contacto prolongado de estudiantes con pacientes ${ }^{1,9,10}$. 
En esta investigación, diseñada para ensayar una experiencia de acompañamiento de un enfermo por estudiantes de medicina, ellos manifestaron no haber experimentado cambios sustanciales en sus conceptos previos con respecto a lo que significa una enfermedad crónica. Sin embargo, los participantes confirmaron que la práctica médica no se restringe a tratar sólo la patología sino que debe asumir también los problemas psicológicos, personales, familiares y sociales que vive el paciente. Es posible que experiencias docentes similares, realizadas con alumnos seleccionados con otros criterios, o con alumnos de cursos superiores, con mayor experiencia, puedan mostrar resultados diferentes.

El grupo de pacientes, constituido en base a invitación no selectiva de sus médicos tratantes, conformó un grupo heterogéneo de enfermos que por esta razón compartieron vivencias personales muy diversas con los estudiantes. Con una selección diferente de enfermos la experiencia de los estudiantes podría cambiar, pero lo esencial del aprendizaje, que es comprender de manera cercana lo que ocurre en el enfermo y su grupo familiar, debería ser equivalente.

Para los alumnos participantes este estudio constituyó una experiencia importante. No sólo conocieron el sufrimiento de algunos pacientes sino que, en otros casos, observaron cómo se pueden adaptar a las condiciones que les exige la enfermedad. Los estudiantes tuvieron la oportunidad de relacionarse de manera directa y prolongada con un enfermo crónico, lo cual les significó además ejercitar formas de comunicación en esta compleja relación. A su vez, vivieron la experiencia de entregar apoyo y afecto al enfermo sólo a cambio de una satisfacción personal, lo cual fue destacado por alumnos y por algunos enfermos. Estos aspectos formativos para adquirir habilidades de comunicación y de apoyo se lograron de manera complementaria a los objetivos primarios planteados para la adquisición de conceptos de enfermedad. De hecho la experiencia, entendida como un contacto humano vivido en un contexto clínico y social, refuerza el aprendizaje sobre la enfermedad, el enfermo y el rol profesional del médico $^{2}$. El aporte del acompañamiento a un enfermo, compartiendo tiempos prolongados con él y conociendo personalmente su entorno social y familiar, posibilita que el estudiante comprenda que la enfermedad ocurre de manera diferente en cada persona y que adquiere consecuencias y significados propios en cada caso, tanto para el enfermo como para su familia. La enfermedad se expresa en cada enfermo de una manera existencial y no sólo biológica, concepto que se busca que los estudiantes adquieran en las asignaturas de humanidades médicas ${ }^{11}$. Programas como el realizado aspiran a que los estudiantes comprendan que los enfermos son personas con historias y experiencias personales y familiares más que sujetos portadores de una enfermedad ${ }^{12,13}$. Este concepto fue fortalecido por este grupo de alumnos, lo cual se demostró cualitativamente en esta investigación.

Los resultados de esta experiencia revelan que ella se constituyó en una oportunidad efectiva de aprendizaje que podría ser parte de la formación de los estudiantes de medicina, con las adaptaciones y cambios que correspondan. Si bien en este grupo de alumnos no se observó un cambio sustancial de su visión previa sobre las consecuencias de la enfermedad, el efecto observado fue que consolidaron sus conceptos teóri$\cos$ y adquirieron una experiencia vivida que fortalece su sensibilidad con la situación de los enfermos. En base a esta experiencia preliminar proponemos que en posibles programas similares la selección de los alumnos participantes sea amplia y que ella se realice preferentemente en cursos más avanzados de la carrera, etapa en la cual los estudiantes podrían profundizar aún más su relación con los enfermos y obtener mayor beneficio mutuo. Por otra parte, los pacientes a quienes se invite a colaborar en proyectos de este tipo deberían tener un diagnóstico relativamente reciente, con enfermedades que sean para ellos un motivo de pérdida de su autovalencia. Finalmente, es preferible realizar el período de acompañamiento a comienzos del año académico, época en que los alumnos tienen más tiempo disponible. 


\section{ReFERENCIAS}

1. Martínez R. The Nature of Illness Experience: A course on Boundaries. Theor Med Bioeth 2002; 23: 259-69.

2. Dornan T, Bundy C. What can experience add to early Medical Education? Consensus survey. BMJ 2004; 329(7470): 834.

3. Hicks LK, Lin Y, Robertson DW, Robinson DL, Woodrow SI. Understanding the Clinical Dilemmas that shape Medical Students' Ethical Development: Questionnaire survey and focus group study. BMJ 2001; 322: 7288.

4. Yedidia MJ, Gillespie CC, Kachur E, Schwartz MD, Ockene J, Chepattis AE et al. Effect of communication: Training on Medical Student Performance. JAMA 2003; 290: 9.

5. Wykurz G, Kelly D. Developing the role of patients as teachers: Literature review. BMJ 2002; 325: 7368 .

6. Rodríguez Gómez G, Gil Flores J, García Jiménez E. Metodología de la Investigación Cualitativa. Ed Aljibe, Biblioteca de Educación. Málaga, España, 1996.
7. Darer JD, Hwang W, Pham HH, Bass EB, Anderson G. More Training needed in Chronic Care: A survey of US Physicians. Acad Med 2004; 79: 541-8.

8. Lempr H, Seale C. The Hidden Curriculum in undergraduate medical education: qualitative study of medical students' perceptions of teaching. BMJ 2004; 329: 770-3.

9. Armstrong E, Parsa-Parsi R. How can Physicians' learning styles drive Educational Planning? Acad Med 2005; 80: 680-4.

10. Bideau M, Guerne Pa, Bianchi MP, Huber P. Benefits of a Programme taking advantage of Patient-instructors to teach and assess Musculoskeletal Skills in Medical Students. Ann Rheum Dis 2006; 65: 1626-30.

11. Gaver A, Borkan JM, Weingarten MA. Illness in context and Families as Teachers: A year-long project for Medical Students. Acad Med 2005; 80: 448-51.

12. Evans M. Reflections on the Humanities in Medical Education. Med Educ 2002; 36: 508-13.

13. Fitzgerald F. An Academic Clinician's Perspective on the Care of the Geriatric Patient. Health Care Anal 2005; 13: 95-100. 